\title{
Fonemas cacuminales procedentes de -LL- en el altoaragonés de época medieval y moderna: indicios a través de las grafías
}

\author{
Jesús VÁZQUEZ OBRADOR ${ }^{1}$ \\ Universidad de Zaragoza \\ vazob@unizar.es
}

\begin{abstract}
RESUMEN
A través del empleo de determinadas grafías como $d, d d, t d, z, z s, t z$ y $t s$ por parte de escribanos del Alto Aragón, podemos deducir la existencia de dos fonemas cacuminales distintos procedentes de -11en el aragonés de áreas geográficas también diferentes del tercio norte la provincia de Huesca: el oclusivo sonoro $/ \mathrm{d} / \mathrm{y}$ el africado sordo $/ \mathrm{t}^{\mathrm{S}} /\left(</ \mathrm{d}^{\mathrm{Z}} /\right)$. Estos, con el paso del tiempo, acabaron por fonologizarse respectivamente bien en el dental oclusivo sordo $/ \mathrm{t} /$, bien en el palatal africado sordo /ĉ/. Con respecto a la época concreta en que se produjo la transformación, no se puede precisar ahora con exactitud, pues es necesario recopilar más testimonios. Es posible, no obstante, que a fines del siglo XVIII ya hubiesen desaparecido los viejos fonemas de las localidades más conservadoras.
\end{abstract}

Palabras clave: aragonés, diacronía, fonemas cacuminales

[Recibido, spetiembre 2010; aprobado, diciembre 2010]

\section{Apical-palatal phonemes derived from -LL- in Mediaeval and Modern High Aragonese: evidence from spelling}

\begin{abstract}
The use of consonants and consonant clusters such as $d, d d, t d, z, z s, t z$ y $t s$ by clerks in the Spanish region of Alto Aragón shows the existence of two different apical palatal phonemes derived from LL- in the varieties of Aragonese spoken in two different areas within the northern third of the Huesca province: voiced plosive $/ \mathrm{d} / /$ and voiceless affricate $/ \mathrm{t}_{\Lambda}^{\mathrm{S}} /\left(</ \mathrm{d}^{\mathrm{Z}} /\right)$. With the pass of time these changed into new phonemes, respectively as: voiceless dental plosive /t/ and voiceless palatal affricate / $\mathrm{c} /$. A specific time for the shift cannot be accurately established from the data now available to us, but it seems highly likely that older phonemes, which were common in the most conservative areas, would have already dropped out of use by the turn of the $18^{\text {th }}$ century.
\end{abstract}

Keywords: Aragonese, diachrony, apical-palatal phonemes

1. Como resulta conocido, la evolución de la líquida geminada -LL- en determinadas áreas pirenaicas y prepirenaicas oscenses en las que el aragonés fue la lengua autóctona se produjo en dos direcciones. Por un lado, el énfasis de su articulación aumentó la aplicación del dorso de la lengua al paladar, de manera que aparecieron

\footnotetext{
${ }^{1}$ Miembro del Grupo de Investigación FILAR (Filología Aragonesa), reconocido por el Gobierno de Aragón y cofinanciado por el Fondo Social europeo.
} 
los fonemas dorsopalatales como $/ \lambda /^{2}$; por otro, la exageración articulatoria se realizó con el ápice, más o menos retroflexo, por lo que surgieron los cacuminales o ápico-palatales. Estos últimos, en la Península Ibérica, quedaron confinados a algunas zonas de nuestro romance así como del astur-leonés; fuera de ella, a algunos dialectos meridionales de Italia y también de Sicilia y Cerdeña ${ }^{3}$.

Centrándonos en el aragonés, parece evidente que esos fonemas de carácter ápico-palatal no alcanzaron el siglo XX, pues ni siquiera el filólogo francés J. J. Saroïhandy, que realizó sus primeras excursiones lingüísticas por el Alto Aragón a partir del año 1896, indica en sus publicaciones algo sobre la existencia de aquellos en las zonas por él visitadas ${ }^{4}$. Deberemos deducir, por tanto, que el citado investigador se encontró ya con los fonemas en los que aquellos habían desembocado -concretamente $/ \mathrm{t} / \mathrm{y} / \hat{\mathrm{c}} /$ para las zonas en las que vamos a centrar nuestro trabajo ${ }^{5}$ ya que si hubiese hallado algún matiz cacuminal a buen seguro que lo habría señalado.

Conocido, pues, el resultado final, es lícito que nosotros nos preguntemos ahora hasta cuándo pudieron estar vigentes, en determinadas áreas dialectales de nuestro romance, los fonemas/sonidos cacuminales a los que me refería arriba. Ciertamente, la respuesta no resulta fácil, y, en cualquier caso, deberá venir de la mano de la documentación escrita, aun cuando ella tampoco nos ayuda demasiado, pues en Aragón, durante el Medioevo, se tardó más tiempo que en otras regiones hispánicas peninsulares en llevar el romance autóctono a la escritura, ya que el latín se mantuvo con gran vitalidad hasta la primera mitad del siglo XIII, siendo la lengua en la que se redactaba la práctica totalidad de los escritos, tanto los de carácter oficial como los privados ${ }^{6}$. Pero además de la dificultad señalada, nos encontramos

\footnotetext{
${ }^{2}$ Resultado común con otras lenguas hispánicas como el castellano y el catalán.

${ }^{3}$ En algunas hablas de esas tres últimas áreas esos fonemas han llegado hasta nuestros días. En el gascón del sur de Francia también hubieron de existir, pero lo que hallamos hoy no son fonemas propiamente ápico-palatales, sino los resultados finales de la evolución de ellos, como en aragonés.

${ }^{4}$ Comenzó sus indagaciones en la localidad ribagorzana de Graus y posteriormente estuvo en otras poblaciones de Sobrarbe y el Viejo Aragón, lo cual le permitió publicar en 1901 un artículo en el que describía de forma global y algo somera las hablas de Ansó, Echo, Bielsa, Gistaín y Benasque (cf. Saroïhandy 1901).

${ }_{5}^{5}$ Prescindo intencionadamente de los resultados $/ \mathrm{z} / \mathrm{y} / \mathrm{s} /$, pues hubieron de ser propios del romance primitivo de buena parte de Ribagorza, como nos muestra su toponimia. También lo hago de / $\mathrm{f} /$, presente en algunas-muy pocas- voces aragonesas, como por ej., el alomorfo ro, ra, ros, ras del artículo determinado en posición intervocálica, empleado en el aragonés de la zona central del tercio norte de la provincia de Huesca, desde el valle de Tena hasta el de Puértolas, y, por el sur, hasta los Somontanos de Huesca y Barbastro. Aparece también en las voces belsetanas er 'él', era 'ella' (pronombres personales) y aquer 'aquel', aquera 'aquella' (demostrativos). De todos modos, no acabo de ver con claridad que la alveolar vibrante simple de estos vocablos del aragonés de Bielsa responda realmente a una solución autóctona en la que se hubiese operado el paso -LL- > / / /.

${ }^{6}$ Es verdad, no obstante, que ya en este tipo de documentación se puede reconocer en ocasiones alguna parcela del romance, pues aparecen insertados, por razones difíciles de precisar (¿descuido,
} 
con otra para nuestro intento de rastrear determinados fenómenos de la lengua autóctona en la documentación, ya que ni siquiera en la scripta romance usada a partir de la segunda mitad del siglo XIII por notarios y escribanos que ejercen su actividad en los valles pirenaicos se refleja fielmente el altoaragonés ahí hablado, pues determinados aspectos de la lengua vernácula eran evitados con asiduidad a la hora de escribir ${ }^{7}$. Y a ellas aún podemos sumar otro inconveniente más, como es que el aragonés desaparezca casi totalmente de la escritura administrativa ya durante la primera década del siglo $\mathrm{XVI}^{8}$. No obstante, y a pesar de todos esos obstáculos, es posible encontrar en la documentación algunos testimonios que nos ayuden a conocer mejor el pasado de nuestra lengua autóctona, y no solo en lo que atañe a aspectos de su léxico, sino también a la morfología e, incluso, a la fonología.

Y precisamente en este último plano lingüístico es en el que me quiero centrar ahora, ya que la lectura de nueva documentación hace que vayamos conociendo más datos que nos confirman la muy probable existencia de dos fonemas cacuminales o ápico-palatales distintos, concretamente, el oclusivo /d्A/ y el africado /d dz/, luego ensordecido /țs/, en el altoaragonés de diferentes áreas geográficas del tercio norte del Alto Aragón, los cuales, con el paso del tiempo, acabaron por fonologizarse respectivamente bien en el dental oclusivo sordo / $t /$, bien en el palatal africado sordo /ĉ/. Con respecto a la época o momento concreto en que se produjo la transformación, no se puede precisar ahora con exactitud, pues es necesario recopilar más testimonios. De todos modos, el hecho de que el P. León Benito Martón, natural de Sallent de Gállego (valle de Tena), escribiese aún en 1760 voces como Castietso [hoy, Castiecho] y Zarrastietso [hoy, Zarrastiecho], hace que sospeche que en esa época todavía se mantendría un fonema de carácter cacuminal procedente de -LL- en el aragonés local y comarcal, por lo que es lícito retrasar hasta finales del siglo XVIII el tiempo en que pudo haberse dado la transformación fonológica y la consiguiente desaparición de, cuando menos, el segundo de esos dos fonemas ápico-palatales a los que aludía antes.

Para defender tal hipótesis me baso en el empleo que hacen de ciertas grafías los escribanos, en determinados vocablos, mayoritariamente topónimos, y en textos de diferentes siglos, pues ellas son las que nos permitirán deducir la existencia de los susodichos fonemas cacuminales y precisar, en la medida de lo posible, su vigencia cronológica.

ignorancia, intencionalidad?), vocablos en los que se pueden percibir fenómenos lingüísticos de la lengua hablada popularmente.

${ }^{7}$ Costumbre que no deja de resultar extraña, si la analizamos desde una óptica propiamente aragonesa.

${ }^{8}$ Así como de los textos literarios, aunque es verdad que a mediados del siglo XVII encontramos varios autores, como Ana Abarca de Bolea, el Vicario de Cariñena, doña Isabel de Rodas y Aráiz, etc., que emplean el aragonés en sus composiciones poéticas en lo que pudo haber sido un intento de dignificar dicha lengua para la literatura. 
Paso, pues, a detallar las que he encontrado en la documentación manejada 9 .

\section{Grafía $d$}

Como se puede comprobar por los datos expuestos a continuación, es usada por escribanos de Jaca y de los valles de su entorno. Algunos de los testimonios de que disponemos, que no agotan, por supuesto, el listado posible, son los siguientes:

1. Agnedera. Muy probablemente, la $-d$ - presente en este nombre de lugar, atestiguado en un documento ${ }^{10}$ del año 867 ( $C D C H$, I: 25 , doc. 7), pero transmitido en una copia del siglo XIII, no representa el fonema dental oclusivo sonoro /d/, sino el cacuminal o ápico-palatal continuador de -LL- ${ }^{11}$. Pienso en esa posibilidad teniendo en cuenta que en un manuscrito posterior, de 1116, se registra el que parece ser el mismo topónimo, si bien se escribe como Agnellera (CS: 55, doc. 29$)^{12}$, y, evidentemente, tanto una forma como la otra se podrían corresponder fonéticamente con el actual Añetera/Ñetera $(\text { Echo })^{13}$, explicable como continuador de una base latina *AGNELLARIA, derivado colectivo en -ARIA del diminutivo AGNĚLLU 'corderito', y este, a su vez, de AGNUS 'cordero, ${ }^{14}$.

2. Badatas (Jaca, 1422: Gómez de Valenzuela 2003a: 47, doc. 2). No hay inconvenientes para suponer que su étimo es el lat. VALLATAS, participio sustantivado del verbo VALLO 'rodear de vallado o empalizada', 'atrincherar'. Se corresponderá, por tanto, con el Batatas, Bachatas o Ballatas de otros puntos altoaragoneses.

${ }^{9}$ Con respecto a la lectura de protocolos notariales (abreviadamente, prot.), advierto que no ha sido ni mucho menos exhaustiva, pues me he limitado a realizar algunas calas entre los numerosísimos (varios miles) conservados en el Archivo Histórico Provincial de Huesca, pertenecientes a los siglos XV-XVIII. Mientras no se indique lo contrario, deberá entenderse que los citados en el trabajo se hallan depositados en el mencionado Archivo.

${ }^{10}$ En el que el conde aragonés Galindo Aznárez realiza diversas donaciones al monasterio de San Pedro de Siresa y señala los límites de la localidad de Echo.

${ }^{11}$ Para el que algún otro escribano de la comarca de Jaca también empleó $d d$ en época coetánea (cf. § 3.4).

${ }^{12}$ En CDCH, I: 146, doc. 119, Durán Gudiol anotó, sin embargo, Agnella al transcribir ese nombre del mismo manuscrito, pero suponemos que será errata.

${ }^{13}$ Hay también Barranco l'Añetera.

${ }^{14}$ No hay inconveniente alguno para suponer que la /t/ moderna del citado topónimo proceda de LL-, por cuanto en la toponimia del valle de Echo (y zonas aledañas) hay muchos ejemplos de dicha evolución e, incluso, queda algún resto en el aragonés hablado, como casieto 'especie de armario para guardar la ropa', 'caseta rústica y pequeña construida en un campo alejado' (cf. también lo señalado en nota 31$)$. 
3. Badato (Echo, 1424: prot. 6.886, ff. 50v, 63v $\left.{ }^{0}\right)^{15}$. Badato [de San Jorge] (Siresa, 1426: prot. 6.886, f. 96r) $^{16}$. Badato [Liso] (Jasa, 1580: prot. 10.100, f. $\left.8 \mathrm{v}^{0}\right)^{17}$. Es término hermano de Batato (Sinués) y de Bachato (cf. $\S$ 6.3). Proviene del lat. VALLATU 'vallado', 'rodeado de empalizada o foso'.

4. Badaton (Urdués, 1457, prot. 6.863, f. $7 \mathrm{r}^{\circ}$ ). Se puede explicar como derivado diminutivo en -ONE de VALLATU.

5. Badi (Echo, 1427: prot. 6.886, f. 102r ${ }^{\circ}$ ); [Cabo de] Badi (ibid., f. $101 \mathrm{v}^{\circ}$ ). Teniendo en cuenta la existencia actual en Echo de los topónimos Batichane, Batimala, así como de La Bati en el vecino Aragüés del Puerto, parece factible aceptar que el registro Badi pudiese remontar al lat. VALLE 'valle'.

6. Betatiedo (Echo, 1559: prot. 6.849 , f. $4 v^{0}$; 1564 : prot. 6.853 , f. $43 v^{0}$ ). Hoy, Betatieto. Proviene del diminutivo VETATĚLlU, formado sobre el participio latino sustantivado VETATU 'lugar vedado'.

7. Biscassieda. Se trata del apellido de una persona de nombre Domingo, que se localiza en un manuscrito de principios del siglo XIV, con la particularidad de que también en él aparece, unas líneas más adelante, la variante Biscassida $\left(C S A F: 215\right.$, doc. 161) ${ }^{18}$. Evidentemente, tal vocablo se corresponderá fonéticamente con el topónimo moderno Biscasillas, nombre de una antigua pardina ya despoblada ubicada en el término de Anzánigo ${ }^{19}$, que muy probablemente es también a la que se refiere el testimonio Bescasella registrado en un documento del año $1191^{20}$, así como el Bescasialla del siglo XIV recogido por Durán Gudiol (1961: 89). La terminación -ialla de este último nos vendría a confirmar que -ieda es realmente la continuadora del sufijo diminutivo latino ÉLLA. Por otra parte, la existencia del mencionado -ieda apunta a que era con

${ }^{15}$ Sin embargo, en 1427 el mismo notario anota Ballato (prot. 6.886, f. $102 \mathrm{r}^{\circ}$ ).

${ }^{16}$ En 1543, empero, se registra Vallato de Sant Jorge (prot. 7.793, f. $43 \mathrm{v}^{\circ}$ ).

${ }_{18}^{17}$ Pero en el f. $4 \mathrm{v}^{\mathrm{o}}$, el mismo notario había apuntado Ballato Liso.

${ }^{18}$ Ahí se recoge la lista de tributos que el monasterio de San Andrés de Fanlo recaudaba en las poblaciones cercanas de Larbesa, Lerés e Ipiés. El contexto es el siguiente: "Item, Domingo Biscassieda, de la estaulia del palaçio, .VI. quartales de trigo treudo [...]. Maria Gronostue et Domingo Biscassida [sic] deven pagar otras .II. galletas". Con respecto al cartulario en que se halla el citado documento cabe señalar que si importante para los historiadores aragoneses ha sido su "reaparición" (estaba en paradero desconocido desde hace unas décadas) no lo es menos para los filólogos, pues ahora se tiene la posibilidad de leer algunos de los documentos copiados en él y que hasta la fecha habían permanecido inéditos, como es el caso del que nos ocupa, y que contienen datos lingüísticos muy interesantes relativos al aragonés medieval.

${ }_{19}^{19}$ Según Ubieto Arteta (1984-1986, III: 1375-1376, s.v. Viscasillas).

20 "damus ad vos Sancio de Leres illam hereditatem de Bescasella cum Benue" (CDCH, II: 449, doc. 462). Ese último nombre se refiere a la localidad hoy llamada Binué, al sudeste de Jaca. 
el fonema cacuminal como se pronunciaba popularmente el topónimo en el aragonés local del siglo XIV, así como en el de lugares vecinos.

8. Codata [do Furno] (Siresa, 1425: prot. 6.886, f. 93v $)^{21}$. Hoy, Cotata. Proviene de COLLATA 'collada'. Es nombre hermano de Cochata (cf. § 6.11).

9. Cuntieda (Urdués, 1457: prot. 6.863, f. 7r $\mathrm{r}^{\circ}$ ). Sospecho que este topónimo se corresponde con el actual Condieta. De ser así, no habría inconvenientes para defender que la terminación -ieda del testimonio del XV procede del sufijo diminutivo latino -ĚLLA.

10. Planiedas (Cenarbe, 1554: prot. 6.846, f. $169 \mathrm{r}^{\circ}$ ). Se trata del moderno Planietas, pronunciado también como Plañetas debido a la palatalización de la $/ \mathrm{n} /$ por influencia de la yod romance del diptongo/ie/. Su étimo es el lat. PLANĚLLAS 'llanuritas'.

11. Rio Mariede, Rigo Mariede (Echo, 1423: prot. 6.886, f. $37 \mathrm{r}^{\circ}$, repetidas ambas formas dos veces). Hoy, Romariete. El segmento -mariete (a diferencia del inicial Ro-, interpretable como síncopa de Rigo 'río') no posee una explicación clara.

12. la Vade (Ulle-Barós, 1441: Gómez de Valenzuela 2007: 111, doc. 32). Hoy, La Bate, cuyo étimo es el lat. VALLE 'valle' (cf. Bache en § 6.4).

13. Si la etimología de Agnedera (cf. arriba) fuese realmente la propuesta, la grafía $-d$ - se convertiría, por ahora, en la primera registrada en nuestro romance para representar el fonema cacuminal continuador de -LL-, adelantándose en unos años a - $d d$ - (cf. § 3.4).

\section{Grafía $d d$}

$\mathrm{Al}$ igual que ocurre con la analizada en el apartado anterior, esta grafía también es usada por escribanos de Jaca y de los valles de su entorno.

1. Baddatun (Urdués ${ }^{22}$, 1543: prot. 7.793 , f. $2 \mathrm{r}^{\mathrm{o}}$ ). Tal vez se trate del Badaton que hemos visto en $\S 2.5$, pero con $u$ en la sílaba final por un error del escribano.

2. Baddichaner, Vaddichaner (Echo, 1541: prot. 7.792, f. $\left.78 \mathrm{r}^{\circ}\right)^{23}$. Hoy, Batichane. Teniendo en cuenta la forma actual (así como los registros citados en nota 22), puede explicarse Baddi- como continuador del lat. VALLE 'valle'.

${ }^{21}$ En 1437, empero, el mismo notario anota Collada del Furno (prot. 6887, f. 20r ${ }^{\circ}$ ).

${ }^{22}$ A lo largo de todo el protocolo, la localidad aparece denominada Ordues. 
3. Coddata Plana (Echo, 1594: Gómez de Valenzuela 2007: 440, doc. 197). Hoy, Cotata Plana. Evidentemente, su étimo será el sintagma latino COLLATA PLANA 'collada llana'.

4. deuaddat. Este vocablo se registra en un manuscrito pinatense que remonta al año $893(C S J P, 32, \text { doc. } 7)^{24}$, si bien se nos ha transmitido en una copia del siglo XII (op. cit., 31). Dicha voz se corresponde con la más usual deualla (devalla) 'baja', 'desciende' (infinitivo deuallar), registrada en diversos textos medievales aragoneses, aunque no de forma abundante. En lo que atañe a su origen, se explicará como un derivado verbal del latín VALLE 'valle' (DECLLC, s.v. vall).

5. Sedde ${ }^{* 25}$. Es el nombre de un puerto de montaña ${ }^{26}$ que aparece mencionado así en ciertos protocolos de notarios que ejercieron su labor en la ciudad de Jaca, fundamentalmente: $1481^{27}$ (Gómez de Valenzuela 2007: 200, doc. 85, en dos ocasiones); $1568^{28}$ (cf. Vázquez 2007: 73, en tres ocasiones); $1570^{29}$ (Vázquez 2007: 73) y $1578^{30}$ (Gómez de Valenzuela 2007: 424, doc. 192).

6. Torrecieddas (Guasa, 1566: prot. 9.309, f. $225 \mathrm{r}^{\circ}$ ). Este topónimo se puede explicar como descendiente de TURRICĚLLAS 'torrecitas', diminutivo en -ĚLLA del latín TǓRRE.

\section{Vaddichaner (vide Baddichaner)}

${ }^{23}$ En 1439, sin embargo, se registra Ballichaner (prot. 6.887, f. 64r ${ }^{\circ}$ ). También en 1468 (prot. 6.865 , f. $116 \mathrm{r}^{\circ}$ ).

${ }^{24}$ Aunque el editor del documento, Antonio Ubieto, escribió - $v$ - he podido comprobar que en el manuscrito original donde se encuentra aquel (Libro gótico o Cartulario de San Juan de la Peña, conservado en la Facultad de Derecho de la Universidad de Zaragoza) hay - $u$-. Aprovecho, de paso, para señalar que en el folio $78 \mathrm{v}^{\circ}$ del citado manuscrito, y en la misma línea en la que aparece la palabra dauaddat, en lugar del vocablo spelunca transcrito por Ubieto lo anotado es spelunzella, con signo de abreviatura de nasal sobre la - $u$-.

${ }^{25} \mathrm{El}$ asterisco colocado detrás de un topónimo indica que este ha sido registrado en textos manuscritos, pero que no ha llegado hasta nuestros días.

${ }^{26}$ Término comunal de algunas de las localidades de los valles de Garcipollera (cuenca del Aragón) y de Acumuer (cuenca del río Gállego), y sobre el que existieron diversas disputas vecinales durante algunos años.

${ }^{27}$ Notario Domingo de Campo (Jaca).

${ }^{28}$ Notario Pedro de Anglada (Jaca).

${ }^{29}$ Notario Miguel de Pardinilla (Jaca).

${ }^{30}$ Notario Pedro de Anglada (Jaca). 
En cuanto a su cronología, esta grafía se registra por vez primera en un documento del año 893, si bien transmitido en una copia del siglo XII. Tarda en reaparecer, pues en los testimonios aquí recogidos no lo hace hasta el año 1481.

\section{Grafía $t d$}

Teniendo en cuenta que este dígrafo aparece en el topónimo Setde (1482), que, sin embargo, el mismo notario (Domingo de Campo [de Jaca]) escribía como Sedde en uno de sus protocolos del año anterior (cf. Vázquez 2007: 72), creo que se puede considerar como una mera variante del grafema $d d$ analizado en el apartado precedente.

5. Por lo que respecta al fonema que se intenta representar mediante las tres grafías analizadas hasta ahora $(d, d d$ y $t d)$, no parece arriesgado suponer que debía de tener una articulación ápico-palatal, probablemente sonora aún en el siglo XVI como cabría deducir del empleo de la grafía $d$, y tal vez con un carácter geminado (/dd d $/$ ) si nos atenemos a los testimonios en que aparecen dos letras. De todos modos, no descarto que ya desde época temprana se tratase de un degeminado oclusivo /d d/ y que la duplicación de la $d$ en la escritura respondiese a un recurso ortográfico de los escribanos para materializar el aspecto cacuminal del fonema, cuya pronunciación se asemeja a la de una geminada al poseer dos momentos articulatorios que hacen que su duración temporal sea un poco más larga que la de otros sonidos.

Dicho fonema estaría vigente en el aragonés hablado popularmente en ciertas áreas pirenaicas y pre-pirenaicas, entre las que se encontrarían casi todo el Campo de Jaca, algunos valles situados al norte (Echo, Aragüés, Borau, Canfranc, Garcipollera), así como otras zonas ubicadas al sur de la primera capital del reino de Aragón, fundamentalmente en el área de la sierra en la que se halla el monasterio de San Juan de la Peña. Nuestra deducción se basa en dos hechos fundamentales: por un lado, que en su toponimia se halle hoy mayoritariamente el resultado $/ \mathrm{t} /<$ LL-; por otro, que han sobrevivido algunos restos léxicos en los que se encuentra la misma solución: casieto 'especie de armario para guardar la ropa', 'caseta rústica y pequeña construida en un campo alejado' ${ }^{31}$, catella 'espacio que queda entre dos casas contiguas', etc.

En cualquier caso, aun cuando el fonema hubiere sido inicialmente geminado, está claro que acabó por degeminarse, perdió su articulación ápico-palatal en beneficio de la dentalidad mediante un adelanto del punto de articulación, así como el

${ }^{31}$ Que, en esta segunda acepción, he atestiguado como casiello en bastantes protocolos de notarios de Echo del siglo XV. 
rasgo de sonoridad ${ }^{32}, \mathrm{y}$, en esas zonas a que me refería arriba, acabó siendo atraído $\mathrm{y}$ asimilado por el oclusivo dental sordo $/ \mathrm{t}^{\beta 3}$, que ya existía en el sistema consonántico aragonés, pero procedente de otros orígenes.

En cuanto al mantenimiento temporal del susodicho fonema ápico-palatal en el aragonés de las áreas mencionadas, creo que pudo haberse dado por lo menos hasta el siglo XVII, pues es lo que cabría deducir del empleo de las grafías analizadas por parte de los escribanos (véase, empero, lo señalado en $\S \S 11.1$ y 15.1).

\section{Grafía $z$}

La encuentro en protocolos redactados por los notarios del Valle de Tena y de Tierra de Biescas. Con ella se intenta representar el fonema continuador (por aquel entonces) de la geminada -LL-, que acabó por desembocar en el palatal africado sordo $/ \hat{\mathrm{c}} /{ }^{34}$ Sus testimonios son:

1. Avezaneto (Pueyo de Tena, 1426: Gómez de Valenzuela 2002: 62, doc. 5) ${ }^{35}$. Hoy, Abechaneto. Proviene del colectivo ABELLANĚTU 'avellanedo'.

2. Bazatarons* (Sallent de Gállego, 1450: prot. 6.998 , ff. $7 \mathrm{v}^{\mathrm{o}}$ y $\left.25 \mathrm{v}^{\mathrm{o}}\right)^{36}$. Es un derivado en -ón de un arcaico y desusado *Bazatar, correspondiente fonético del cast. valladar, siendo, por tanto, derivado del latín VALLATU 'vallado', 'rodeado de empalizada o foso'.

3. Bazato (Sallent de Gállego, 1425: prot. 7.000, f. 14v ). Bazato [de las Articas]* (Sallent, 1427: prot. 6.999, f. 20v $\mathrm{v}^{\mathrm{o}}$ ). Se corresponde con el moderno Bachato y proviene del lat. VALLATU 'vallado', 'rodeado de empalizada o foso'.

\footnotetext{
${ }^{32}$ Más detalles sobre la evolución de -LL- hasta llegar a los resultados presentes en el altoaragonés moderno pueden verse en Vázquez Obrador 1993: 398-400, § 4.

${ }^{33}$ Tal solución, empero, no es exclusiva de las citadas áreas altoaragonesas, pues se halla también en el Sobrarbe (valles de Broto-Torla, etc.) e, incluso, algún resto se encuentra en el valle de Benasque, como por ej., Batisielles y Estatats (Benasque).

${ }^{34}$ En Tena es esa la solución característica. En Tierra de Biescas lo es de todas las localidades de las zonas norte y central.

${ }^{35}$ No obstante, en protocolos de años posteriores se registra Abellaneto. Así, 1482 (prot. 6.963, f. $7 \mathrm{r}^{\circ}$ ) y 1486 (prot. 6.966 , f. $5 \mathrm{r}^{\circ}$ ). En 1562 aparece Abellanedo (prot. 7.852 , f. $2 \mathrm{r}^{\circ}$ y ss.), pero $-\mathrm{y}$ esto es lo interesante - en el índice general del mismo protocolo, donde se recogen los extractos de cada una de las escrituras, se atestigua como Abezaneto.

${ }^{36}$ En el f. $7 \mathrm{v}^{\circ}$ se atestigua la variante Ballatarons.
} 
4. Baze (Sandiniés, 1426: prot. 7.803, f. 6v $\mathrm{v}^{\circ}$ ). Baze (Sallent, 1427: prot. 6.999, f. $15 v^{037} ; 1450$ : prot. 6.998 , f. $\left.24 v^{0}\right)$. Hoy, Bache. Su étimo es el lat. VALLE 'valle'.

5. Bazella (Oz de Tena, 1499: prot. 6.975, f. 14vº). Bazelha* (Sallent, 1431: prot. 7.768, f. $3 \mathrm{r}^{\circ}$ ). Bazella, Bazellya y Bazellyas (Panticosa, 1480: prot. 6.961, ff. $24 \mathrm{v}^{\mathrm{o}}$ y $25 \mathrm{r}^{\circ}$ ). Reaparece Bazella en 1500 (prot. 6.976, f. $17 \mathrm{r}^{\circ}$ ) y, Bazellas en 1506 (prot. 6.981, f. $13 \mathrm{r}^{\circ}$ ). Bazella (Lanuza, 1506: prot. 6.981, f. 4r ${ }^{\circ}$ ). Bazellas (E1 Pueyo de Tena, 1426: Gómez de Valenzuela 2002: 62, doc. 5). Se trata de los actuales Bachella y Bachellas. Provienen del diminutivo VALLǏCŬLA(S) 'vallejos'.

6. Bazillaço (Tramacastilla de Tena, 1498: prot. 6.974 , f. $8 \mathrm{v}^{\circ}$ ). Actualmente, Bachillazo. Se puede explicar, en última instancia, como derivado despectivo de VALLE (con cambio de género) o de VALLU 'foso', 'parapeto'.

7. Bazimanya (Panticosa, 1516: Gómez de Valenzuela 2006: 219, doc. 153); 1575 (prot. 6.921, f. 31 $v^{\circ}$ ). Hoy, Bachimaña. Su étimo es el lat. VALLE MAGNA 'valle grande'.

8. Berrjezo* (Escarrilla, 1425: prot. $\left.7000, \mathrm{f} .12 \mathrm{v}^{0}\right)^{38}$. La terminación -jezo es la continuadora del sufijo diminutivo latino -ĚLLU.

9. Castezillo (Pueyo de Tena, 1426: Gómez de Valenzuela 2002: 62, doc. 5). Castezilho* (Panticosa, 1450: prot. 6.998, f. 3r $\mathrm{r}^{\circ}$ ). Su étimo será el despectivo latino CASTELLǏCǓLU 'castillejo'.

10. Cletiaza (Aso de Sobremonte, 1483: prot. 6964, f. $23 \mathrm{v}^{\circ}$ ). Hoy Cletiacha. Desciende de *CLETĔLLA, diminutivo del celtismo CLETA 'cancilla'.

11. Cozata (Sandiniés, 1426: prot. 7.803, f. 6vº). Cozata (Sallent de Gállego, 1450: prot. 6.998, f. $12 \mathrm{v}^{\mathrm{o}}$ ). Cozatas (Panticosa, 1563: Gómez-Navarro 2002: 154, doc. 33); 1605 (Gómez de Valenzuela 2006: 366, doc. 257). Se trata de los modernos Cochata y Cochatas, derivados del lat. CŏLlATA 'collada'. Hay también Cozata ra Metulla (Panticosa, 1427: Gómez de Valenzuela 2002: 71, doc. 8), denominado hoy Cochata ra Metula.

\footnotetext{
${ }^{37}$ Pero un año después, 1428, el mismo notario escribe Balle (prot. 6.997, f. $12 \mathrm{r}^{\mathrm{o}}$ ).

${ }^{38}$ No obstante, en el folio $12 \mathrm{r}^{\circ}$ se atestigua en dos ocasiones la variante Berriello.
} 
12. Cozatiaza* (Panticosa, 1480: prot. 6.961, f. $25 \mathrm{r}^{\circ}$ ). Se explica como descendiente del diminutivo COLLATĚLLA 'collada pequeña'.

13. Es Betatiaz ${ }^{39}$ (Tramacastilla de Tena, 1498: prot. 6.974, f. $8 v^{0}$ ). Es el moderno Esbetatiach. Provendrá de ǏLLOS VETATĚLL(O)S 'los vedadillos'. 14. Es Cuazs $^{40}$ (Panticosa, 1487: prot. 6.967, f. 17r ${ }^{\circ}$ ). Se atestigua también como Es Quazs $^{41}$ (1498: prot. 6.974, f. 12ro ) y Scuaz (1506: prot. 6.981, f. 5v $\mathrm{v}^{\circ}$. En 1532 hay Scuez (Gómez de Valenzuela 1992: 93, doc. 50) Se trata del actual Escuach. Su étimo es el sintagma ǏLLOS CŎLL(O)S 'los cuellos'.

15. Spatriaz* (Panticosa, 1489: prot. 6.969, f. $13 \mathrm{v}^{\mathrm{o}}$ ). De haber llegado a nuestros días, probablemente lo hubiese hecho bajo la forma Espatriás, registrada en otros puntos oscenses. Proviene de ÍLLOS PRATĔLl(O)S 'los pradillos'.

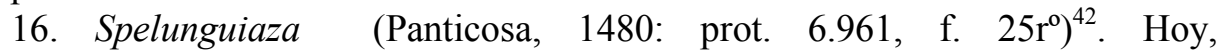
Espelunguiacha. Es un derivado en -iacha ( $<$ lat. -ĚLLA) del apelativo espelunga 'cueva'.

17. Staziello (Panticosa, 1605: Gómez de Valenzuela 2006: 366, doc. 257). Actualmente, Estatiecho. Provendrá del diminutivo *STALLĚLLU, formado sobre una base céltica *STALLOM 'estancia', 'establo' (véase el siguiente).

18. Stazo (Sallent de Gállego, 1431: prot. 7.768, f. $34 r^{\circ}$ ). Stazo (Panticosa, 1480: prot. 6.961 , f. $\left.25 \mathrm{r}^{\circ}\right)^{43}$. Actualmente, Estacho, cuyo étimo parece ser un céltico *STALLOM 'estancia para recoger el ganado', 'especie de establo' (DECLLC, s.v. estall). Se registran también Stazo* (Pueyo de Tena, 1497: prot. 6.973, f. 16r ${ }^{\circ}$ ); Stazo Calbet* (Sallent, 1428: prot. 6.997, f. 12ro ); Stazo

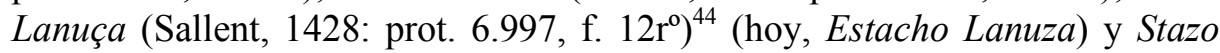
Mediano* (Tramacastilla, 1501: Gómez de Valenzuela 2006: 179, doc. 118), registrado también este último con grafía $-t z-(\mathrm{cf} . \S 8.8)$.

\footnotetext{
${ }^{39}$ Aparece en el sintagma "Campo des Betatiaz".

${ }^{40} \mathrm{El}$ artículo en el manuscrito está unido a la preposición en: "enes Cuazs".

${ }^{41}$ El contexto es: "as Quazs, cerqua Fayezo", donde as responderá a la contracción de la preposición $a$ con el artículo de plural es: $a$ es $>a$ 's.

${ }^{42}$ En 1489 y 1495 aparece escrito como Spelungujella (prot. 6.969 , f. $14 \mathrm{r}^{\mathrm{o}}$; prot. 6.972 , f. $40 \mathrm{v}^{\circ}$ ).

${ }^{43}$ No obstante, en 1431 aparecía como Stalho (prot. 7.768 , f. $38 \mathrm{v}^{\circ}$ ). Años después se registra Estallo (1487: prot. 6.967, f. $16 \mathrm{v}^{\circ}$ ) y Stallo (1488: prot. 6.968, f. 3r $\mathrm{r}^{\circ}$; 1497: prot. 6.973 , f. $6 \mathrm{v}^{\circ}$ ).

${ }^{44}$ No obstante, en 1443, hay Stallyo Lanuça (prot. 7.818, f. $6 \mathrm{r}^{\circ}$ ), al igual que en 1495 (prot. 6.972, f. $\left.18 \mathrm{r}^{\mathrm{o}}\right)$.
} 
19. Stazs (Panticosa, 1480: prot. 6.961, f. $25 \mathrm{r}^{\circ}$ ). Se registra también como Staz en 1491 (prot. 6.971, f. $4 \mathrm{r}^{\circ}$ ) y 1506 (prot. 6.981, f. 14v $\mathrm{v}^{\mathrm{O}}$ ); Estaz ${ }^{45}$ en 1491 (prot. 6.971, f.4v $\mathrm{v}^{\mathrm{O}}$ ). Estaz (Oz de Tena, 1563: Gómez de Valenzuela 2002: 162, doc. 40). Es el moderno Sestás $(<*$ Sestazs $<*$ Es Estazs $<*$ Es Estazos $)$, cuya etimología la constituirá el sintagma ǏLLOS *STALLOS.

20. Fayezo (Panticosa, 1498: prot. 6.974, f. 12r ${ }^{\circ}$ ). En la actualidad, Fayecho. Provendrá de FAGĚLLU, diminutivo de FAGU 'haya'. Se atestigua también con grafía -tz- (cf. § 8.9).

21. Fontiazas (Panticosa, 1506: prot. 6.981, f. $\left.12 \mathrm{v}^{\mathrm{o}}\right)^{46}$. Deriva del diminutivo FONTĚLLAS 'fuentecillas'.

22. Fornjaz (Panticosa, 1489: prot. 6.969, f. $14 \mathrm{r}^{\mathrm{o}}$ ); 1495 (prot. 6.972 , f. $40 \mathrm{v}^{\circ}$ ), 1506 (prot. 6.981, f. 14r); Forniaz: 1563 (Gómez-Navarro 2002: 154, doc. 33). Forniazs (Pueyo de Tena, 1482: prot. 6.963, f. 26vº ). Fornjazs (Panticosa, 1488: prot. 6.968, f. $\left.11 \mathrm{v}^{\circ}\right)$. Es el moderno Esforroniás, que procede del lat. ǏLLOS FŬRNĚLL(O)S 'los pequeños hornos [de cal o carbón vegetal]'. Se registra también Forniatz (cf. $\S 8.10$ ).

23. Lacuniazas (Panticosa, 1480: prot. 6.961, f. $25 \mathrm{r}^{047} ; 1567$ : Gómez-Navarro 2002: 174, doc. 43). Hoy Lacuniachas, cuyo étimo es el diminutivo LACUNĚLLAS 'lagunillas'.

24. Lacunieza (Sandiniés, 1426: prot. 7.803, f. $\left.7 \mathrm{r}^{\circ}\right)$. Moderno Lacuniacha $(<$ LACUNĚLLA 'lagunita').

25. Lopiezo (Tramacastilla de Tena). Se atestigua en el año 1440 como apodo de un habitante de la citada localidad: "Lop de Lop alias Lopiezo" (Gómez de Valenzuela 2006: 108 , doc. 37) ${ }^{48}$. Evidentemente, se trata de un derivado diminutivo en -iezo ( $<$ lat. -ĚLLU) del antropónimo Lopo o Lupo, que equivaldrá, por tanto, al apellido acastellanado Lopillo.

\footnotetext{
${ }^{45}$ Esta última forma en el contexto "Collada d'Estaz".

${ }^{46}$ Quizá se trate del mismo topónimo que en 1499 se mencionaba como Fontiellas (prot. 6.975, f. $\left.14 \mathrm{v}^{\mathrm{o}}\right)$.

${ }^{47}$ Pero se halla Lacuniellas en 1482 (prot. 6.963, f. 19r $\mathrm{r}^{\circ}$ ), 1484 (prot. 6.965, f. 16vo ), 1497 (prot. 6.973 , f. $\left.3 \mathrm{r}^{\circ}\right), 1499$ (prot. 6.975 , f. $1 \mathrm{v}^{0}$ ) y 1506 (prot. 6.981 , f. $\left.13 \mathrm{v}^{\circ}\right)$.

${ }^{48} \mathrm{Sin}$ embargo, cuatro años después, en 1444, aparece cambiado en Lopico: "Lop de Lop alias Lopico" (op. cit., 118, doc. 48); y dos años más tarde (1446) se registra Lopielho: Lop de Lop alias Lopielho" (op. cit., 127, doc. 60).
} 
26. Petrosiaza (Búbal, 1484: prot. 6.965, f. 13vº). Hoy, Petrusiacha. Procederá de PETROSĚLLA 'pedrosilla'.

27. Planieza (Sallent de Gállego, 1450: prot. 6.998, f. 25v ; 1495: Gómez de Valenzuela 2002: 115 , doc. 25$)^{49}$. Moderno Planieche. Su étimo, el diminutivo PLANĚLLA 'llanurita'.

28. Planiezo* (Panticosa, 1480: prot. 6.961, f. 25r ${ }^{\circ}$ ). Planiezo (Biescas, 1534: prot. 7.061, f. 41v $)^{50}$. Es el moderno Planiecho. Proviene de PLANĚLLU 'llano pequeño'.

29. Portiaza (Biescas, 1534: prot. 7.061, f. $\left.41 \mathrm{v}^{0}\right)^{51}$. Hoy, Portiacha $(<$ PORTĚLLA 'puerta pequeña', 'paso estrecho').

30. Sarrambuzo* (Panticosa, 1480: prot. 6.961, f. $25 \mathrm{r}^{\circ}$ ). Si tenemos en cuenta que en Sallent, Biescas y Aso de Sobremonte existe Zarrambucho no parece arriesgado suponer que un topónimo igual pudo haber existido en Panticosa a juzgar por el testimonio citado ${ }^{52}$.

31. Sarratiezo (Panticosa, 1430: Gómez de Valenzuela 2006: 90, doc. 21). Actualmente, Sarratiecho. Sarratiezo (Sallent, 1533: Gómez-Navarro 2002: 150, doc. 31), que, posiblemente, se corresponderá con el conocido hoy como Zarrastiecho. Se trata de descendientes del diminutivo SERRATĚLlU 'pequeña loma entre barrancos'.

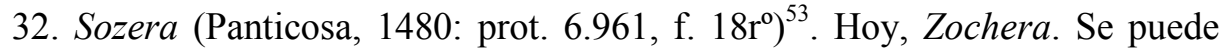
explicar como derivado de una base *SULLLARIA, colectivo en -ARIA formado sobre *SŬLLE (véase el siguiente).

33. sozes*. El testimonio de este vocablo es muy interesante, pues no se trata de un topónimo sino de un apelativo equivalente del moderno zolles (es decir, 'pocilgas'), que estaría vigente aún en el año en que se atestigua, en el arago-

\footnotetext{
${ }^{49}$ No obstante, en 1428 se atestiguaba Planielha (prot. 6.997, f. $12 \mathrm{r}^{\circ}$ ), y años más tarde, en 1495, Planielhas (prot. 6.972, f. $18 \mathrm{r}^{\circ}$ ).

${ }^{50} \mathrm{Sin}$ embargo, en 1481 se anotaba Planiello/Planjello (prot. 6.962 , fols. $15 \mathrm{r}^{\circ}$ y $16 \mathrm{r}^{\circ}$ ), lo mismo que en 1482 (prot. 6.963 , f. $29 \mathrm{v}^{\circ}$ ) y 1545 (prot. 6.907 , f. $5 \mathrm{r}^{\circ}$ ).

${ }_{52}^{51}$ Pero había Portiella en 1481 (prot. 6.962, f. $16 \mathrm{r}^{\circ}$ ).

${ }^{52}$ Para aspectos relacionados con su posible etimología, aún insegura, cf. Vázquez Obrador 2008: 172-175.

${ }^{53}$ No obstante, en 1491 aparece Sollera (prot. 6.971, f. 3ro ), lo mismo que en 1499 (prot. 6.975, f. $\left.21 v^{0}\right)$.
} 
nés del Valle de Tena, pues con el susodicho valor aparece en un documento de 1519 (Gómez de Valenzuela 1992: 34, doc. 12) ${ }^{54}$. Como he señalado en el párrafo anterior, su étimo será una base latino-vulgar *SŬLLE $(<* \text { SŬTLE })^{55}$.

34. Sozs (Panticosa, 1487: prot. 6.967, f. $16 \mathrm{r}^{\circ}$ ). Hay $\operatorname{Soz}$ en el f. $16 \mathrm{v}^{\mathrm{o}}$, forma que vuelve a aparecer en 1495 (prot. 6.972, f. 41 $\mathrm{r}^{\circ}$ ). Provendrá del plural *SŬLL(E)S (véase la entrada precedente). Estos nombres podrían corresponderse con el moderno Zoche (cf. § 9.8), aun cuando del primer testimonio escrito se desprende que lo esperado hoy sería más bien una forma en plural. Quizás se trate de dos topónimos diferentes.

35. Trongazar (Sallent de Gállego, 1431: prot. 7.768, f. 3ro ; 1518: GómezNavarro 2002: 26, doc. 136). Hoy, Trongachar. Quizá provenga de un colectivo latino-vulgar *TRǓNCALLARE, derivado de TRǓNCU 'tronco'.

36. Val de Biazas (Tramacastilla de Tena, 1498: prot. 6.974, f. 9r ${ }^{\circ}$ ). En la actualidad, Baldibiachas. Podría interpretarse el segmento -biachas como un diminutivo en -ĔLLA, del latín VIA 'camino'.

37. Una vez vistos todos los testimonios anteriores, se puede observar que los primeros datan del año 1425 (Bazato y Berrjezo), mientras que el último es de 1605 (Staziello).

Con respecto al valor fonético del fonema cacuminal que se intenta representar con la letra $z$, hemos de desechar que fuese idéntico al de /z/, es decir, el dentoalveolar africado sonoro [dz], o, incluso, al del sordo / $/ \hat{\mathrm{s}} /$, o sea, [ts], existentes, por lo menos el último, en aragonés medieval ${ }^{56}$ y operativos aún en el castellano del siglo XV y comienzos del XVI, ya que, de haberse pronunciado como [ds] o [ts], nuestro fonema cacuminal se hubiese visto arrastrado también hacia el interdental fricativo sordo /९/, como ocurrió con aquellos, al igual que en castellano ${ }^{57}, \mathrm{y}$, sin embargo, ello no

\footnotetext{
${ }^{54}$ El contexto es el siguiente: «que ningun vezino ni habitador de los ditos lugares no sian gosados tener puerco ninguno en todos los terminos de los ditos lugares menos de sortillas sino que se los tengan ensarados en sus casas o sozes en la pena susodita [...]» (loc. cit.).

${ }_{55}$ Para todo lo relacionado con la etimología posible del aragonés zolle 'pocilga', véanse DCECH, s.v. zolle, y DECLLC, s.v. soll.

${ }^{56}$ De todos modos, y aunque ahora no puedo entrar en detalles para intentar demostrarlo, encuentro indicios para sospechar que en el altoaragonés occidental y central, en el que lo característico es la conservación de las oclusivas sordas intervocálicas etimológicas -P-, -T- y -C- (seguida de /a/, /o/, $/ \mathrm{u} /$ ), no llegó a existir el sonoro / /.

${ }^{57}$ En esta lengua, ya en la segunda mitad del siglo XVI ambos habían confluido en el fonema interdental fricativo sordo $/ \Im /$, generalizándose tal pronunciación a comienzos del XVII. Esa misma cronología podría defenderse para la aparición de dicho fonema en aragonés (pero no descarto que
} 
fue así, pues no hemos encontrado ni un solo caso en aragonés en el que haya / $/$ / como resultado de la evolución de -LL- (véanse más detalles en $\S 11)^{58}$.

\section{Grafía zs}

Sólo la he atestiguado, por ahora, en el topónimo Estazso (Panticosa, 1479: prot. 6.959, f. 39v ${ }^{\circ}$ ), que, no obstante, se registra también como Stazo (cf. § 6.18). Resulta interesante esta grafía por cuanto la presencia de $-s$ - parece que nos está indicando que el fonema que quiere encarnar poseía un carácter sibilante, lo cual vendría a ser confirmado por las que vamos a ver en los dos apartados siguientes.

\section{Grafía $t z$}

Atestiguada en:

1. Batze (Escarrilla, 1489: prot. 7.800, f. $9 \mathrm{r}^{\mathrm{0}}$ [dos veces]). Hoy, Bache (cf. § 6.4).

2. Betatiatz (Tramacastilla de Tena, 1486: prot. 7.800 , ff. $4 \mathrm{v}^{\mathrm{o}}$ y $6 \mathrm{r}^{\circ}$ ). Es el actual Esbetatiach, del que hemos tratado en $\S 6.13$.

3. Castietzo. Es este el apellido de un habitante de El Pueyo de Tena, de nombre Domingo, atestiguado en 1429 (Gómez de Valenzuela 1992: 43, doc. 19). Tal forma se corresponde con el término Castiecho (es decir, 'castillo'), topónimo localizado en El Pueyo de Tena y Sallent de Gállego (cf. § 9.2). En 1435 y 1438 aún se sigue empleando ese mismo significante (op. cit.: 76, doc. 45; 87, doc. 52; 92, doc. 56), si bien posteriormente es sustituido por el "más normalizado" Castiello. Su étimo es el lat. CASTĚLLU 'castillo', 'lugar fortificado'.

4. Cotzatiatzas (Sandiniés, 1496: prot. 7.800 , f. $24 \mathrm{r}^{\circ}$ ). Muy probablemente este topónimo será el que ha llegado a nuestros días bajo la forma Cotatiecha/Cotatiacha. Se corresponde con el Cozatiaza registrado en $\S 6.12$ (véase).

5. Cutza (Sandiniés, 1496: prot. 7.800, f. 24r ${ }^{\circ}$ ). En la actualidad, La Cucha. No es seguro que estemos ante un nombre en el que la grafía -tz- sea la continuadora de la geminada -LL-, pues su origen etimológico resulta algo incierto.

fuese anterior). Lo que no creo, sin embargo, es que, como defienden algunos estudiosos, la presencia de tal fonema en nuestra lengua se deba a una propagación desde la castellana.

${ }^{58}$ En otro orden de cosas, cabe señalar que en los protocolos que hemos analizado nunca se emplea la grafía $c$ para escribir el fonema cacuminal del que venimos hablando. 
6. Entrecastietzo (Torla, 1715: Gómez-Navarro 2002: 339, doc. 335, dos veces). Hoy, Andescastieto/Endescastieto ${ }^{59}$. Parece provenir de ǏNTER CASTĚLLU 'entre (el) castillo, ${ }^{60}$.

7. Estatzo (Lanuza, 1480: Gómez-Navarro 2002: 116, doc. 16). Véase, no obstante, Estazo en $\S 6.18$.

8. Estatzo Mediano* (Tramacatilla de Tena, 1486: prot. 7.800, f. $4 \mathrm{r}^{\circ}$ ). En realidad, el topónimo aparece escrito Estatza Mediano, pero evidentemente la - $a$ final de Estatza ha de ser un lapsus del escribano en lugar de -o, como nos demostraría el adjetivo que le sigue, que está en masculino, así como otro registro citado al final del $\S 6.18$.

9. Fayetzo (Escarrilla, 1497: prot. 7.800, f. 15r ${ }^{\circ}$ ). Actualmente, Fayecho (cf. $\S$ $6.20)$.

10. Forniatz (Tramacastilla de Tena, 1486: prot. 7.800 , ff. $4 \mathrm{r}^{\mathrm{o}}$ y $5 \mathrm{v}^{\mathrm{o}}$ ). Hoy, Esforroniás (más detalles en $§ 6.22$ ).

11. Iboncietzo* (Tramacastilla de Tena, 1486: prot. 7.800, ff. $4 \mathrm{r}^{\mathrm{0}}$ [dos veces] y $5 \mathrm{v}^{\circ}$, con la particularidad de que en este mismo folio la segunda vez que aparece lo hace bajo la forma Ibonciello). Evidentemente, de haber llegado a nuestros días, el significante moderno sería Ibonciecho, topónimo existente en Sallent de Gállego. Se trata de un diminutivo en -ciecho $(<-$-CÉLLU) del apelativo aragonés ibón 'lago de montaña de pequeñas dimensiones'.

12. Setze* (Garcipollera-Valle de Acumuer, $1545^{61}$ : Gómez de Valenzuela 2003a: 136, doc. 35). Pero téngase en cuenta que también se registra como Sedde (§ 3.3) y Seche $(\S 10.5)$.

\footnotetext{
${ }^{59}$ Como es conocido, en la zona de Torla-Broto el fonema final en que ha desembocado la evolución de la geminada -LL- ha sido /t/. De todos modos, como el notario que redacta el documento es Miguel Matías Guillén, de Panticosa, no sé si la grafía - $t z$ - usada en el topónimo deberá considerarse realmente representativa del fonema cacuminal imperante en el aragonés de Torla en esas fechas (quizás aún un ápico-palatal /d d/), por cuanto podría tratarse en realidad de una adaptación de aquel a la fonética del aragonés tensino, máxime teniendo en cuenta que en el mismo documento se cita Puybieto, topónimo que podría indicar que el fonema cacuminal ya había comenzado a articularse como /t/, si su étimo fuere un sintagma PǑDIU BĚLLU 'otero bonito' o, tal vez, 'otero de buena vista'.

${ }^{60}$ No obstante, como con la preposición İNTER esperaríamos que el sustantivo que le sigue estuviese más bien en plural, no descarto que el significado hubiese sido 'en medio del castillo'.

${ }^{61}$ Notario Martín de Exea (Jaca).
} 
Como se comprueba por los ejemplos citados, esta grafía se atestigua en protocolos de notarios tensinos, si bien su aparición no resulta muy abundante. Su primer testimonio data del año 1429 (Castietzo) mientras que el último de los registrados aquí es de 1715 (Entrecastietzo).

\section{Grafía ts}

Se atestigua en:

1. Badietso (Oz de Tena, 1698: Gómez de Valenzuela 1995: 311, doc. 159). Hoy, Badiecho. Proviene del diminutivo VADĚLLU 'vado pequeño'.

2. Castietso (Sallent, 1760: Martón: 76-77). Hoy, Castiecho (véase $\S 8.1$ ).

3. Cutsietso (Búbal-Piedrafita-Saqués, 1633: Gómez de Valenzuela 2006: 422, doc. 297). Se corresponde con el moderno Cochecho(s), cuyo étimo será el lat. CŎLLĚLLU 'cuellecito'.

4. Escuats (Tramacastilla de Tena, 1550: Gómez de Valenzuela 2007: 364, doc. 173; se registra en tres ocasiones, pero un poco más adelante aparece escrito Escuach (loc. cit., 365). Esquets (Pueyo de Tena, 1644: Gómez de Valenzuela 1995: 154 y 155, doc. 72; ibid., 161, doc. 75); 1668: op. cit., 247, doc. 126). Es el moderno Escuach, para cuyo origen véase $\S 6.14$.

\section{Estats (véase Sestats más abajo).}

6. Planietsa (Sallent de Gállego, 1616: Gómez-Navarro 2002: 228, doc. 64). Hoy, Planieche (cf. Planieza en $\S 6.27$ ).

7. Sestats (Oz de Tena, 1698: Gómez de Valenzuela 1995: 303, doc. 159, repetido en tres ocasiones, si bien en una cuarta aparece Estats). Hoy, Sestás (cf. § 6.19).

8. Sotse (Panticosa, 1669: Gómez de Valenzuela 2002: 301, doc. 91). Hoy, Zoche. Para su étimo, cf. sozes (§ 6.33).

9. Zarrastietso (Sallent de Gállego, 1760: Martón: 74). Hoy, Zarrastiecho (cf. $\S 6.31)$.

Como se puede observar, es grafema algo tardío pues su primer registro en los ejemplos aquí recogidos data de 1550 (Escuats); los últimos, de 1760 (Castietso, Zarrastietso). 


\section{Grafía ch}

La emplean los notarios de localidades de la cuenca alta del río Gállego ${ }^{62}$. En los ejemplos de que disponemos su primera aparición en un topónimo para representar el sonido continuador de la geminada -LL- se produce en el año 1470, si bien lo más frecuente durante los siglos XV y XVI es el grafema $z$, como ya hemos visto.

1. Arratiecho* (Sallent de Gállego [?] $]^{63}$, 1533: Gómez de Valenzuela 2002: 142, doc. 34). Procederá de *RIATTĚLLU 'riachuelo'.

2. Clusiecho (Biescas, 1640: Gómez de Valenzuela 2003b: 114, doc. 24). Su étimo será el lat. CLŪSĚLLU o CLAUSĚLLU 'cerrado pequeño'.

3. Espelunciecha (Sallent, 1470: Gómez de Valenzuela 2002: 97, doc. 16). Procede del diminutivo SPELUNCĚLLA 'cueva pequeña'.

4. Estacho (Panticosa, 1570: Gómez de Valenzuela 1992: 190-191, doc. 106). Para su etimología, cf. $\S 6.18$.

5. Seche (Garcipollera-Valle de Acumuer, 1479: Vázquez 2007: 73 [dos veces]). También en 1484 (Gómez de Valenzuela 2007: 212-215, doc. 92 [cinco repeticiones]), y 1486 (Vázquez 2007: 74 [siete veces]).

11. Con respecto a las grafías $z, z s, t z$, $t s$ y $c h(\S \S 6-10)$, constatamos que son usadas por escribanos de poblaciones ubicadas en la cuenca alta del río Gállego, es decir, del valle de Tena y de la Tierra de Biescas.

En lo atingente al fonema que representaban (o querían representar), si nos fijamos especialmente en $z s, t z$ y $t s$, podemos deducir con una cierta fiabilidad que debía de poseer un carácter africado, a juzgar por la presencia de la $s$. Por descontado que dicho rasgo también existiría en los casos en los que aparece el grafema $z$, que, por otra parte, es el de mayor vigor durante los siglos XV y XVI. Probablemente, pues, el fonema cacuminal usado inicialmente sería el africado sonoro $/ \mathrm{d} \mathrm{Z} /$, pronto ensordecido $(/ \mathrm{t} \mathrm{S} /)^{64}$, que con el paso del tiempo los hablantes irían transformando al cambiar su articulación cacuminal más o menos retroflexa por otra más plenamente palatal y africada, resultando así el sonido /ĉ/ el continuador de la palatalidad originaria, tal y como comprobamos por algunos apelativos como cuacha 'especie de collera del yugo', gricha 'especie de langosta', grichón 'gri-

\footnotetext{
${ }^{62}$ Como ya señalé al principio del apartado 6, en los valles de Tena, Sobremonte y Acumuer, así como en buena parte de Tierra de Biescas, el resultado final de la evolución de -LL- ha sido precisamente el fonema palatal africado sordo $/ \hat{\mathrm{c}} /$.

${ }^{63}$ Hoy no existe en esta localidad lugar alguno llamado con ese nombre; sí, en cambio, en Biescas.

${ }^{64}$ Quizás ya a comienzos del siglo XV.
} 
llo', 'saltamontes', tacho 'brote que sale en el troncho de la col', etc., que han llegado hasta nuestros días, y también por la toponimia de las zonas arriba mencionadas.

Que el fonema vigente en esos siglos no sería el palatal africado sordo /ĉ/ nos lo demostraría el que todavía en 1760 una persona culta como el Padre Martón escribía Castietso y Zarrastietso. Ahora bien, que estaría muy próximo a aquel en su articulación lo indicaría el hecho de que la grafía $c h$ ya aparezca usada, aunque de forma minoritaria, en algún topónimo a partir del último tercio del siglo XV (cf. $\S 10)$.

\section{Otras grafías: $s$ y $s s$}

En algunas ocasiones encuentro también las grafías $-s$ - y $-s s$ - utilizadas por escribanos del valle de Tena, sobre todo de la segunda mitad de los siglos XVI y XVII. No obstante, en los nombres en los que se atestiguan no podemos considerarlas representativas del fonema alveolar fricativo sordo /s/, sino meras variantes ocasionales del fonema cacuminal del que venimos hablando, pues en todos los casos (menos en Esferronias), los topónimos en los que se registran han llegado a nuestros días con el sonido palatal africado sordo /ĉ/. Algunos ejemplos son:1. Abessanal (Pueyo de Tena, 1628: Vázquez 2004: 188, § 1.12.1). Hoy, Abechanar < lat. ABELLANARE 'avellanar'.

2. Abessaneto (Pueyo de Tena, 1628: Vázquez 2004: 188, § 1.12.1). Véase Abezaneto $(\S 6.1)$.

3. Bassato (Panticosa, 1628: Vázquez 2004: 188, § 1.12.1). Hoy, Bachato (cf. $\S 6.3)$.

4. Castiesso (Pueyo de Tena, 1564: Gómez-Navarro 2002: doc. 35, p. 160). Actualmente, Castiecho (vide Castietzo y Castietso: $\S \S 8.1$ y 9.1).

5. Cossata (Oz de Tena, 1628: Vázquez 2004: 188, § 1.12.1). Cosatta la Metula (Panticosa, 1696: Gómez-Navarro 2002: doc. 92, p. 298). Hoy, Cochata y Cochata ra Metula (cf. 6.11).

6. Cuesso* (1628: Vázquez 2004: 188, § 1.12.1). Aun cuando no ha llegado hasta hoy, su resultado final hubiese sido Cuecho ( $<$ lat. CŎLLU), como en otros lugares tensinos.

7. Escuas (Pueyo de Tena, 1564: Gómez-Navarro 2002: 160, doc. 35; 1617: ibíd., 233, doc. 67). Esquas (Tramacastilla de Tena, 1646: Gómez de Valenzuela 1995: 179-180, doc. 83). Se trata del actual Escuach (cf. §§ 6.14 y 9.14). 
8. Esferronias (Panticosa, 1567: Gómez-Navarro 2002: 174, doc. 43). Hoy, Esforroniás (cf. § 6.22).

9. Estassiesso (Panticosa, 1628: Vázquez 2004: 188, § 1.12.1), registrado también como Estatiesso (1625: Gómez de Valenzuela 1995: 106, doc. 42). Es el moderno Estatiecho (cf. § 6.17).

10. Estasso (Panticosa, 1628: Vázquez 2004: 188, § 1.12.1). Estaso (Sallent, 1644: Gómez de Valenzuela, 2006, 474, doc. 333). Hoy, Estacho (cf. § 6.18).

11. Gargandiasa (Panticosa, 1605: Gómez de Valenzuela 2006: 366, doc. 257). Es el moderno Gargandiacha, diminutivo en -iacha ( $<$ lat. -ĚLLA) de garganda 'garganta'.

12. Vadiesso (Oz de Tena, 1628: Vázquez 2004: 188, § 1.12.1). Hoy, Badiecho (cf. § 9.1).

13. La Vasse (Sallent de Gállego, 1602: Gómez de Valenzuela 2002: 201, doc. 54). Hoy, A Bache (cf. § 6.4).

14. Carranbuso (Sallent, 1628: Gómez de Valenzuela 1995: 116, doc. 47). Carrambuso en 1656 (Gómez de Valenzuela 2006: 521, doc. 359) y 1658 (Gómez de Valenzuela 2006: doc. 525, 363). También Carrambusso en ese mismo documento y año (loc. cit.). Hoy, Zarrambucho (cf. § 6.30).

\section{A modo de conclusión}

13. Ante los ejemplos citados en los párrafos 2 a 10, se observa que el grafema más antiguo con el que se intenta representar un fonema cacuminal procedente de LL- es $d$, registrado en un documento del año 867, pero transmitido en una copia del siglo XIII. Pocos años después aparece $d d$, anotado en un texto de 893, pero que nos ha llegado también en copia tardía (siglo XII). Como ambos, pues, los conocemos por traslados de siglos posteriores, no se puede saber con certeza si tales grafías estaban o no en los documentos originales. Por consiguiente, creo que, por cuestión de método, dichos años no se deberían tomar exactamente como los primeros para el aragonés en que se registran grafías representativas de los fonemas cacuminales de que venimos hablando ${ }^{65}$.

${ }^{65}$ De todos modos, independientemente de que se hallasen o no en ellos, no dudo de que a finales del siglo IX la cacuminalización de -LL- ya se estaría produciendo en el altoaragonés de diversas comarcas oscenses. 
14. Por otra parte, parece lícito deducir la existencia de dos resultados cacuminales diferentes en época medieval y moderna para dos zonas geográficas también distintas. Así, en Jaca y buena parte de su comarca existiría un fonema ápico-palatal oclusivo, inicialmente sonoro ${ }^{66}, / \mathrm{dd} / \mathrm{\alpha} / \mathrm{o} / \mathrm{d} /$, que tras sufrir ensordecimiento y perder el rasgo de la ápico-palatalidad, más o menos retroflexa, adelantó su lugar de articulación y acabó siendo atraído y asimilado por el oclusivo dental sordo /t/, que ya existía en el sistema consonántico aragonés, pero procedente de otros orígenes: T-, -T-, -PT-, etc.

Sin embargo, en la cuenca alta del río Gállego, situada al este de la jaquesa, existiría inicialmente un fonema ápico-palatal sonoro pero africado $/ \mathrm{d}^{\mathrm{Z}} /$, luego ensordecido, $/ \mathrm{t} \mathrm{S} /$, que acabaría por convertirse en plenamente palatal al ser atraído hacia /ĉ/, existente ya en el sistema aragonés, pero de origen distinto.

Vendría a confirmar nuestra hipótesis el hecho de que notarios de Jaca, al referirse a un determinado lugar, común a los valles de Garcipollera y Acumuer, lo escriban (y nombren) como Sedde y Setde (cf. $\S \S 3.5$ y 4), mientras que los de Tierra de Biescas lo hagan como Setze y Seche (cf. $\S 8.4$ y 10.5).

15. Evidentemente, resultan necesarias la lectura y edición de nuevos protocolos de los siglos XIV-XVIII, no sólo de los escribanos de Jaca y alrededores, sino también del resto de todo el Alto Aragón occidental y central, para poder detectar así, a través de las grafías, la existencia de esos fonemas cacuminales procedentes de -LL- en otras zonas altoaragonesas distintas a las aquí mencionadas. El seguimiento ortográfico deberá hacerse fundamentalmente sobre los topónimos, pues en la scripta aragonesa utilizada por nuestros notarios resultará mucho más difícil — por no decir imposible - rastrear registros claramente populares que se aparten de lo que para ellos debería constituir una "norma" culta del aragonés escrito.

También habrá que fijarse en el momento en que grafías específicas como $d$, $d d$, $t d$, por una parte, y $z$, $z s$, ts $y t z$, por otra, comienzan a ser sustituidas, respectivamente, por $t$ y por $c h$, pues ello nos permitiría establecer alguna hipótesis aceptable sobre la cronología en la que pudieron desaparecer del aragonés hablado los viejos fonemas cacuminales de los que venimos tratando. Solo así se podrá precisar con rigor el proceso del cambio fonológico que acabó con ellos y que no tuvo por qué tener idéntica secuencia cronológica en todas las zonas, ya que en algunas (Valle de Tena) pudo haberse mantenido por más tiempo que en otras (Jaca y alrededores) una articulación de carácter ápico-palatal (cf. § 11.1). De todos modos, es probable que a fines del siglo XVIII tal articulación

\footnotetext{
${ }^{66}$ Posiblemente hasta finales del siglo XV. En el XVI encontramos la grafía - $t d-$, que me hace sospechar que tal vez en esa época se estaba produciendo la pérdida de la sonoridad.
} 
hubiese desaparecido ya incluso de los lugares más conservadores, aunque eso solo lo podremos corroborar con más datos.

\section{Bibliografía}

CDCH (I y II): Antonio Durán Gudiol, Colección diplomática de la Catedral de Huesca, 2 vols., Zaragoza: I, 1965; II, 1969.

CS: Antonio Ubieto Arteta, Cartulario de Siresa. Zaragoza: Anubar, 1986, $2^{\mathrm{a}} \mathrm{ed}$.

CSAF: Carlos Laliena Corbera y Eric Knibbs, El Cartulario del monasterio aragonés de San Andrés de Fanlo (siglos X-XIII). Zaragoza: Departamento de Historia Medieval, Ciencias y Técnicas Historiográficas y Estudios Árabes e Islámicos (Universidad de Zaragoza), 2007.

CSJP: Antonio Ubieto Arteta, Cartulario de San Juan de la Peña. Valencia: Anubar, vol. I, 1962.

DCECH: Joan Corominas y José Antonio Pascual, Diccionario crítico etimológico castellano e hispánico. Madrid: Gredos, 6 vols. 1980 y ss.

DECLLC: Joan Coromines, Diccionari etimologic $i$ complementari de la llengua catalana. Barcelona: Curial Edicions, 9 vols., 1980 y ss.

DURÁN GUDIOL, Antonio (1961): "Geografía medieval de los obispados de Jaca y Huesca”. Argensola 45-46: 1-105.

GómeZ DE VALENZUElA, Manuel (1992): Documentos del Valle de Tena (siglo XVI). Zaragoza: Real Sociedad Económica Aragonesa de Amigos del País.

- (1995): Documentos del Valle de Tena (siglo XVII). Zaragoza: Real Sociedad Económica Aragonesa de Amigos del País.

- (2002): Testamentos del Valle de Tena (1424-1730). Zaragoza: El Justicia de Aragón.

- (2003a): Capitulaciones matrimoniales de Jaca (1420-1791). Zaragoza: El Justicia de Aragón.

- (2003b): Capitulaciones matrimoniales y firmas de dote en el Alto Gállego (1428-1805). Zaragoza: El Justicia de Aragón.

- (2006): Diplomatario tensino (1315-1700). Zaragoza: Real Sociedad Económica Aragonesa de Amigos del País.

- (2007): Documentos sobre ganadería altoaragonesa y pirenaica (siglos XV y $X V I)$. Zaragoza: El Justicia de Aragón.

GómeZ de VAlenzuela, Manuel, y Ana NAVARro Soto (2002): Capitulaciones matrimoniales y firmas de dote en el Valle de Tena (1426-1803). Zaragoza: El Justicia de Aragón.

MARTÓN, Fray León Benito (1760): Sallent, cabeza de el Valle de Tena, sus antiguedades y varones insignes que ha tenido en armas y letras. Pamplona. Hay edición facsímile: Madrid, 1982.

SAROÏHANDY, Jean J. (1901): "Mission de M. Saroïhandy en Espagne", Annuaire de l'École Practique des Hautes Études, 106-118. Se publicó traducido ("In- 
forme del Sr. Saroïhandy"), con un prólogo de Joaquín Costa titulado "Dialectos aragoneses" en la Revista de Aragón, (1902): 644-654. Hay reimpresión de este último artículo en Archivo de Filología Aragonesa, 61-62 (2005-2006): 363-375.

UBieto ARTETA, Antonio (1984-1986): Historia de Aragón. Los pueblos y los despoblados. Zaragoza: Anubar, 3 vols. (I, 1984; II, 1985; III, 1986).

VÁZQUEZ OBRADOR, Jesús (1993): "Soluciones romances de la geminada -LL- en la toponimia de Sobremonte, Ribera de Biescas y Sobrepuerto (Huesca). Intento de explicación fonético-fonológica". Anuario de Estudios Filológicos 16: 391-415.

- (2004): "Notas sobre aragonesismos atestiguados en un documento notarial tensino del año 1628 y en una copia posterior". Alazet 16: 181-246.

- (2007): "Un testimonio medieval antiguo de la cacuminalización de -LL- en aragonés". De Lingva Aragonensi 3: 67-76.

- (2008), Toponimia de Aso, Yosa y Betés (Sobremonte, Alto Gállego). Zaragoza: Prensas Universitarias de Zaragoza-Xordica Editorial. 